\title{
Composição e riqueza de peixes em diferentes corpos d'água no parque nacional das Araucárias, Sul do Brasil
}

O conhecimento das espécies de um determinado ambiente é base para futuros estudos ecológicos, bem como para o auxílio no manejo e conservação de áreas prioritárias. Para tal, esse estudo objetivou inventaria a composição de espécies de peixes em diferentes ambientes aquáticos no Parque Nacional das Araucárias (PNA), Santa Catarina. Foram avaliados 10 pontos amostrais com características distintas (riachos, lago de reservatório, açude e rios de maior porte) ao longo da área do Parque. Para a amostragem diferentes artes de pesca foram empregadas em cinco coletas com periodicidade trimestral. Um total de 1.022 exemplares foi registrado, distribuídos em 22 espécies, sete famílias e quatro ordens, das quais Siluriformes apresentou a maior riqueza para a área de estudo. Astyanax scabripinnis, Oligosarcus brevioris, Hoplias malabaricus e Rhamdia quelen apresentaram a maior amplitude de distribuição, enquanto as demais ficaram restritas a um ou poucos ambientes. Ainda, destaca-se a presença de Crenicichla empheres, espécie considerada endêmica do rio Chapecó, além de Cyprinus carpio espécies exótica, que foi registrada em um açude no interior do Parque. Ademais, por meio da curva de acumulação, foi possível inferir que as maiores parcelas das espécies presentes na área do PNA foram registradas, haja vista a tendência da curva a atingir a assíntota. Por fim, destaca-se a ocorrência de Crenicichla lepidota, Pareiorhaphis vestigipinnis e Pareiorhaphis steindachneri, para as quais não havia ainda, registros para a bacia do rio Chapecó. Ressalta-se que a presença de algumas espécies possivelmente esteja associada a determinadas características estruturais e ambientais encontradas na área do Parque, reforçando a importância de tais áreas para a manutenção da biota, tal como os peixes.

\section{Composition and wealth of fish in different water bodies in the national park of Araucárias, South of Brazil}

\begin{abstract}
The knowledge of the species of a given environment is the basis for future ecological studies, as well as for the assistance in the management and conservation of priority areas. For this, this study aimed to invent the composition of fish species in different aquatic environments in the Araucarias National Park, Santa Catarina state. Ten sample points with distinct characteristics (streams, reservoir lake, pond and larger rivers) were evaluated along the Park area. For the sampling, different fishing gears were used in five collections with quarterly frequency. A total of 1,022 specimens were recorded, distributed in 22 species, seven families and four orders, of which Siluriformes presented the greatest wealth for the study area. Astyanax scabripinnis, Oligosarcus brevioris, Hoplias malabaricus and Rhamdia quelen presented the greatest range of distribution, while the others were restricted to one or a few environments. Also, the presence of Crenicichla empheres, a species considered endemic to the Chapecó River, besides Cyprinus carpio, exotic species, was recorded in a pond inside the Park. Moreover, through the accumulation curve, it was possible to infer that the largest plots of the species present in the NAP area were recorded, due to the tendency of the curve to reach the asymptote. Finally, we highlight the occurrence of Crenicichla lepidota, Pareiorhaphis vestigipinnis and Pareiorhaphis steindachneri, for which there were not yet records for the Chapecó river basin. It should be noted that the presence of some species is possibly associated with certain structural and environmental characteristics found in the Park area, reinforcing the importance of such areas for the maintenance of the biota, such as fish.
\end{abstract}

Keywords: Astyanax Scabripinnis; Oligosarcus Brevioris; Endemic Species; Streams; Conservation Unit.

Topic: Organização da Biodiversidade

Reviewed anonymously in the process of blind peer.

Jerri Andre Berto

Universidade Comunitária da Região de Chapecó, Brasil http://lattes.cnpq.br/6314005812497200

jerri_berto@yahoo.com.br

\section{Criatiano Ilha}

Universidade Comunitária da Região de Chapecó, Brasil

cristiano ilh@hotmail.com

Gilza Maria de Souza-Franco (iD

Universidade Federal da Fronteira Sul, Brasil

http://lattes.cnpq.br/4018616229163111

http://orcid.org/0000-0002-5023-3211

gilza.biologa@gmail.com
Received: 10/04/2018

Approved: 24/05/2018

\section{Referencing this:}

BERTO, J. A.; ILHA, C.; SOUZA-FRANCO, G. M.. Composição e riqueza de peixes em diferentes corpos d'água no parque nacional das Araucárias, Sul do Brasil. Revista Ibero Americana de Ciências Ambientais, v.9, n.4, p.125-137, 2018. DOI: http://doi.org/10.6008/CBPC2179-6858.2018.004.0010 


\section{INTRODUÇÃO}

O levantamento de espécies de um determinado grupo é considerado a pedra fundamental para a elaboração condizente de estudos e projetos de manejo de uma área ou região (CASATTI et al., 2001). Levando em consideração a ictiofauna, Schaefer (1998) lembra que o avanço para sua compreensão depende do inventário biótico de áreas pobremente amostradas, tais como pequenos riachos e ribeirões, a fim de identificar a diversidade existente, possíveis novas espécies, bem como endemismos ou aquelas sob algum risco de extinção. Além disso, conhecer a composição da comunidade de peixes de uma região é primordial para realizar avaliações quanto a alterações ambientais e fundamentar medidas que venham a minimizar possíveis impactos registrados (HICKSON et al., 1995).

Apesar de muitas áreas ainda não terem sido efetivamente estudadas, o Brasil é considerado o país com a maior diversidade íctica do mundo (BUCKUP et al. 2007), contudo, acredita-se que um número significativo de espécies ainda permaneçam desconhecidas ou pouco estudadas (KAUFMANN et al., 2007). Ciente da forte pressão que os remanescentes florestais e cursos hídricos têm sofrido ao longo das últimas décadas, é sabido que muitas destas espécies podem ter desaparecido mesmo antes de serem descritas e estudas. Dentre as causas para tais eventos, a destruição e alteração do meio ambiente pela ação humana (e. g. desmatamento, introdução de espécies exóticas, construção de barragens), é reconhecida como o principal fator para o desaparecimento de espécies, independente do grupo, seja local ou não (CETRA et al., 2010).

Reconhecido como um ambiente altamente impactado e que constantemente sofre pressões externas, o bioma Mata Atlântica está inserido entre os estados do Rio Grande do Sul e Rio Grande do Norte. Conforme Barreto et al. (2005), neste bioma os pequenos riachos exercem importantes funções ecológicas, os quais apresentam por característica, vazão reduzida e alta sensibilidade a alterações e degradação ambiental, o que tem resultado nas últimas duas décadas a uma corrida na busca de informações que possam contribuir com a compreensão do funcionamento ecológico destes sistemas aquáticos.

O conhecimento das espécies de peixes em regiões pouco pesquisadas, como riachos e pequenos rios, estão entre os estudos prioritários para que se tenham informações que subsidiem medidas de preservação da fauna de peixes (REIS et al., 2003). Para tanto, este trabalho buscou inventariar a composição de espécies de peixes em diferentes cursos hídricos no Parque Nacional das Araucárias (PNA), pertencente a bacia hidrográfica do rio Chapecó.

\section{MATERIAIS E MÉTODOS}

\section{Área de Estudo}

Criado no ano de 2005, o Parque Nacional das Araucárias possui aproximadamente 13.000 hectares e está localizado entre os municípios de Ponte Serra e Passos Maia, no estado de Santa Catarina (Figura 1), sendo que, a rede hidrológica faz parte da bacia hidrográfica do rio Chapecó (MMA, 2010). Segundo a divisão das regiões hidrográficas no estado de Santa Catarina, a bacia do rio Chapecó está inserida na região 
hidrográfica RH2 - Meio Oeste e faz parte da bacia do rio Uruguai (SANTA CATARINA, 2007). O Parque Nacional das Araucárias está localizado na porção alta da bacia do rio Chapecó, onde são encontradas as nascentes dos principais rios que percorrem a área do parque (e. g. rio Chapecó, Chapecozinho e rio do Mato), além de diversas nascentes e pequenos afluentes (MMA, 2010).

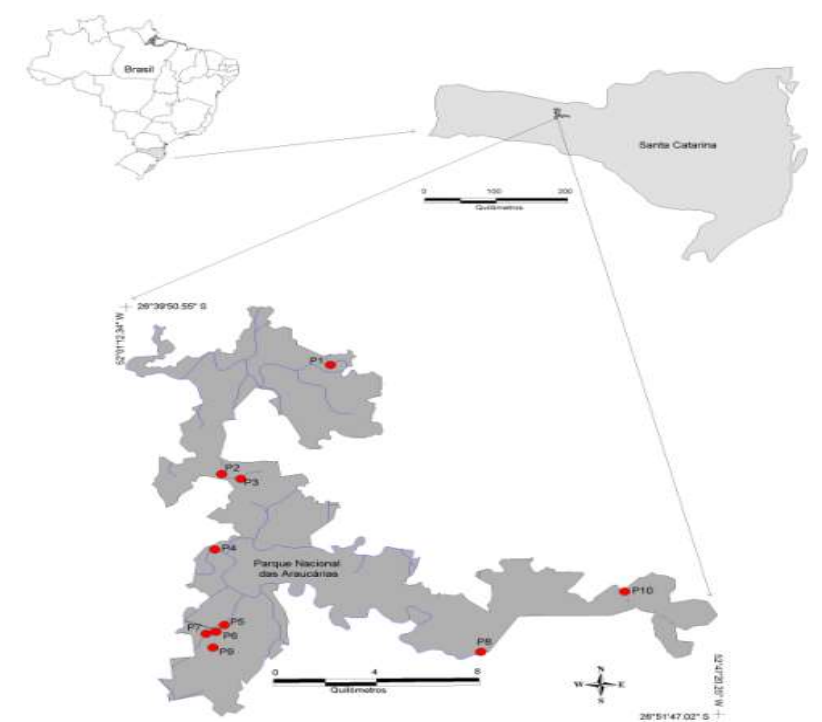

Figura 1: Mapa com localização do PNA e dos pontos amostrais: P1 - Rio Chapecó (RCH), P2 - Riacho Ameixeira (RA), P3 - Riacho dos Morcegos, P4 - Rio Chapecozinho (RCHZ), P5 - Riacho Caratuva Jusante (CJ), P6 - Barramento Caratuva (BC), P7 - Riacho Caratuva Montante (CM), P8 - Rio do Mato (RMT), P9 - Açude Caratuva (AÇC) e P10 Açude Adami (AÇA).

Conforme Klein (1978) a região apresenta como formação vegetacional a Floresta Ombrófila Mista, caracterizada por uma mata branca, onde o pinheiro-brasileiro Araucaria angustifolia surge como uma árvore emergente, não apresentando uma grande densidade e se mostrando em manchas isoladas na floresta subtropical do rio Uruguai.

De acordo com MMA (2010) a área do parque está localizada em uma zona de transição climática, do clima subtropical para o tropical, onde sofre a influência das massas de ar quente oriundas da região central da América do Sul. Estas, ao encontrar as massas de ar frio vindas de regiões mais ao sul, proporciona a ocorrência de chuvas frontais, normalmente nos períodos de primavera e outono. Quanto ao regime pluvial, a área apresenta características próprias de uma região de transição, onde a precipitação total média anual é de $1.970 \mathrm{~mm}$, variando entre um máximo de $3.000 \mathrm{~mm}$ e um mínimo $1.150 \mathrm{~mm}$, onde os meses de outono e inverno são os que mais apresentam excessos hídricos mensais (MMA, 2010). Dessa forma o clima predominante do Parque Nacional das Araucárias, segundo a classificação de Köppen, é temperado chuvoso de ambiente úmido, considerado um clima frio, com temperatura média anual inferior a $18^{\circ} \mathrm{C}(\mathrm{MMA}, 2010)$.

Os ambientes amostrados estão distribuídos ao longo da abrangência da área do parque, onde apresentam ambientes com características distintas, conforme descrito por MMA (2010) (Tabela 1).

Tabela 1: Caracterização das regiões estudadas, com as distintas estações amostrais e suas referências geográficas.

Floresta em estágio secundário avançado de regeneração, lavoura e lagos artificiais. 


\begin{tabular}{|c|c|}
\hline \multirow{2}{*}{\multicolumn{2}{|c|}{$\begin{array}{l}\text { Açude Caratuva } \\
\text { Região do Rio do Mato }\end{array}$}} \\
\hline & \\
\hline \multicolumn{2}{|c|}{ Floresta em estágio inicial, médio e avançado de regeneração, além de áreas de silviculturas. } \\
\hline Rio do Mato & $-2649^{\prime} 07.14379^{\prime \prime} /-5158^{\prime} 57.43969^{\prime \prime}$ \\
\hline Açude Adami & $-2648^{\prime} 11.94494^{\prime \prime} /-5149^{\prime} 31.13981^{\prime \prime}$ \\
\hline
\end{tabular}

Região do riacho dos Morcegos

Floresta em estágio médio e avançado de regeneração com presença de animais domésticos de grande e pequeno porte.

\begin{tabular}{l|l} 
Riacho dos Morcegos & -26 44' 51.78247'/-51 58' 32.15106"
\end{tabular}

\begin{tabular}{l|l} 
Riacho Ameixeira & -26 44' 43.32269”/-51 58' 59.20070"
\end{tabular}

Região do Rio Chapecozinho

Floresta em estágio médio e avançado de regeneração; presença de gado e áreas de silvicultura

\begin{tabular}{l|l} 
Rio Chapecozinho & -26 46' 54.97010"/-51 59' 10.00062"
\end{tabular}

Região do rio Chapecó

Floresta em estágio médio de regeneração com presença de animais domésticos de grande e pequeno porte.

Rio Chapecó

$-2641^{\prime} 33.76545^{\prime \prime} /-5156^{\prime} 24.04855^{\prime \prime}$

\section{Amostragem}

Para o levantamento da fauna de peixes foram realizadas cinco coletas com periodicidade trimestral de julho de 2011 a julho de 2012, as quais estavam autorizadas pelo SISBIO/ICMBio/MMA, inscrição número 27584.1 emitida em 22 de julho de 2011. Com o intuito de contemplar a maior parte da assembleia de peixes, tanto para espécies com hábitos noturnos como para os de hábito diurno, diferentes artes de pesca foram empregadas, sendo elas: redes de espera, espinhel, peneirão, tarrafa, picaré e pesca com linha de mão.

As redes de espera apresentavam $10 \mathrm{~m}$ de comprimento e diferentes malhas $(1,2,2,3,4,5$ e $7 \mathrm{~cm}$ entre nós). $O$ espinhel utilizado continha $15 \mathrm{~m}$ com 10 anzóis de tamanho 4/0. Estas duas artes de pesca permaneceram expostas $24 \mathrm{~h}$ em cada ponto amostral com revisões a cada oito horas. Para o peneirão, picaré e pesca com linha de mão empregou-se uma hora de esforço amostral por unidade de coleta. Já para a tarrafa, duas malhas diferentes foram utilizadas, sendo 1,5 e $3 \mathrm{~cm}$ entre nós. Para cada uma das tarrafas, vinte arremessos em cada unidade de coleta foram realizados.

\section{Processamento do material biológico}

Conforme descrito por Zaniboni et al. (2004), todos os exemplares foram fixados em formol a $10 \%$ e posteriormente transferidos para álcool a $70 \%$. Os espécimes capturados foram identificados com base em guias de campo de peixes da Mata Atlântica (OYAKAWA et al., 2006; MENEZES et al., 2007; GRAÇA et al., 2007) e da bacia do alto rio Uruguai (ZANIBONI et al., 2004), além de consultas a especialistas e chaves de identificação. Os exemplares testemunhos estão depositados no Laboratório de Ecologia da Unochapecó e na Coleção Ictiológica do Nupélia da Universidade Estadual de Maringá.

\section{Análise de dados}

A partir da matriz de presença e ausência de espécies, contabilizou-se a riqueza específica entre os pontos amostrados e a distribuição íctica na área do PNA, para analisar as diferenças em relação à riqueza e eficiência amostral de espécies coletadas e ao longo das campanhas foi realizada curva de rarefação de espécies com o programa PAST (PAleontological STatistic). Conforme Gotelli et al. (2001) esta análise é realizada partindo-se da permutação aleatória de todos os indivíduos capturados. Cada ponto gerado na 
curva representa a média dessas permutações. A análise de rarefação gera o número de espécies esperado para a curva de acumulação de espécies, confeccionada com o total de espécies coletadas durante as amostragens.

\section{RESULTADOS}

A fauna de peixes nos corpos d’água do Parque Nacional da Araucárias, no período de estudo, foi composta de 1.022 indivíduos, distribuídos em 22 espécies (Figura 2) pertencentes a sete famílias e quatro ordens (Tabela 2). A ordem Siluriformes apresentou maior riqueza com onze espécies, seguido de Characiformes e Perciformes com cinco espécies cada, sendo que juntas perfazem 95,45\% da riqueza total amostrada. A ordem Cypriniformes foi a que apresentou a menor representatividade, com apenas uma espécie (4,55\%) (Figura 3).

Dentre as espécies, foram capturadas três de ocorrência não citada para a bacia do rio Chapecó, sendo elas Crenicichla lepidota e os cascudos Pareiorhamphis vestigipinnis e Pareiorhamphis steindachneri. Também foi registrado Crenicichla empheres, endêmica do rio Chapecó. Na região da fazenda Caratuva, a qual faz parte do referido Parque, em um reservatório artificial foram capturados sete indivíduos de Cyprinus carpio, reconhecida como espécie exótica.

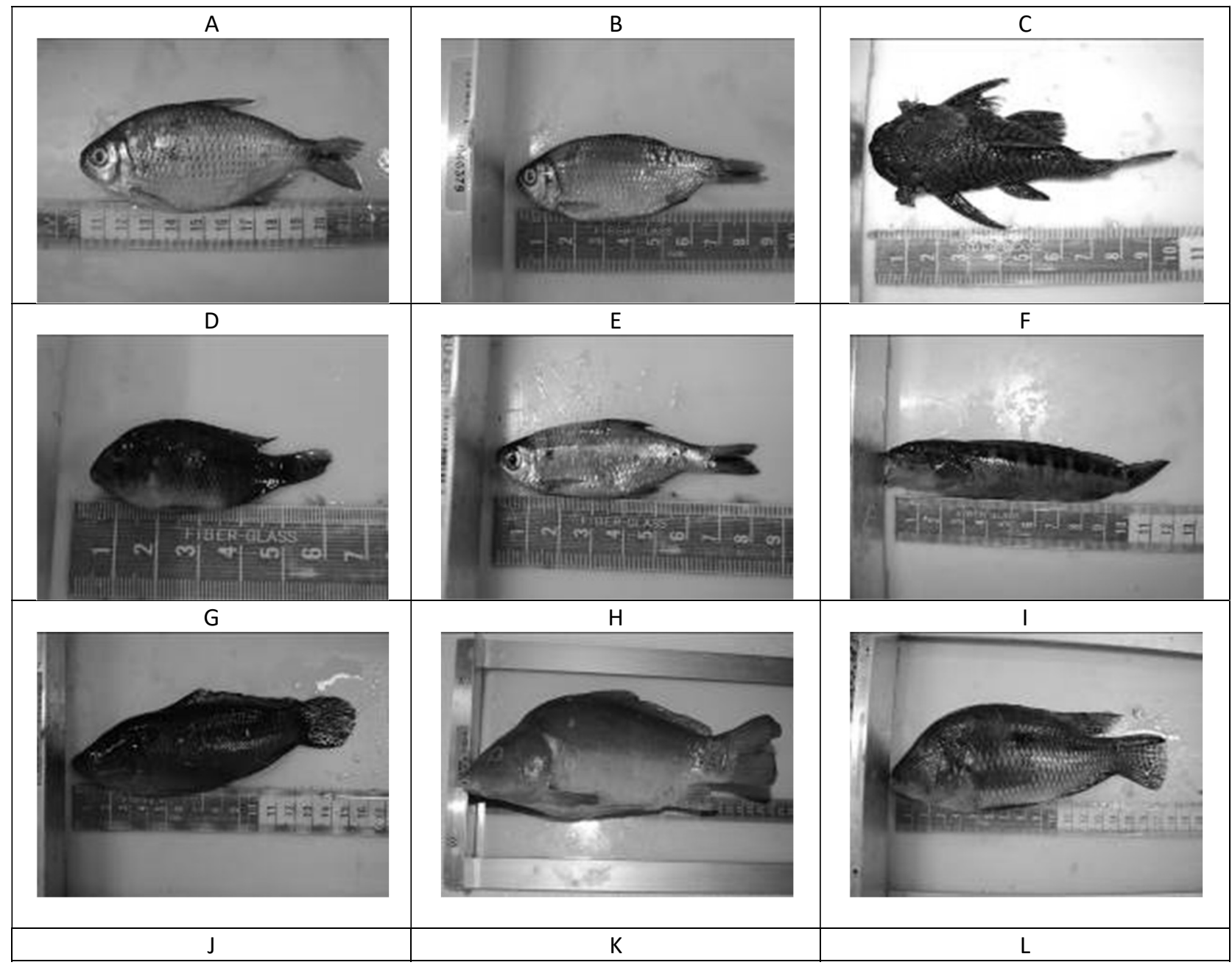




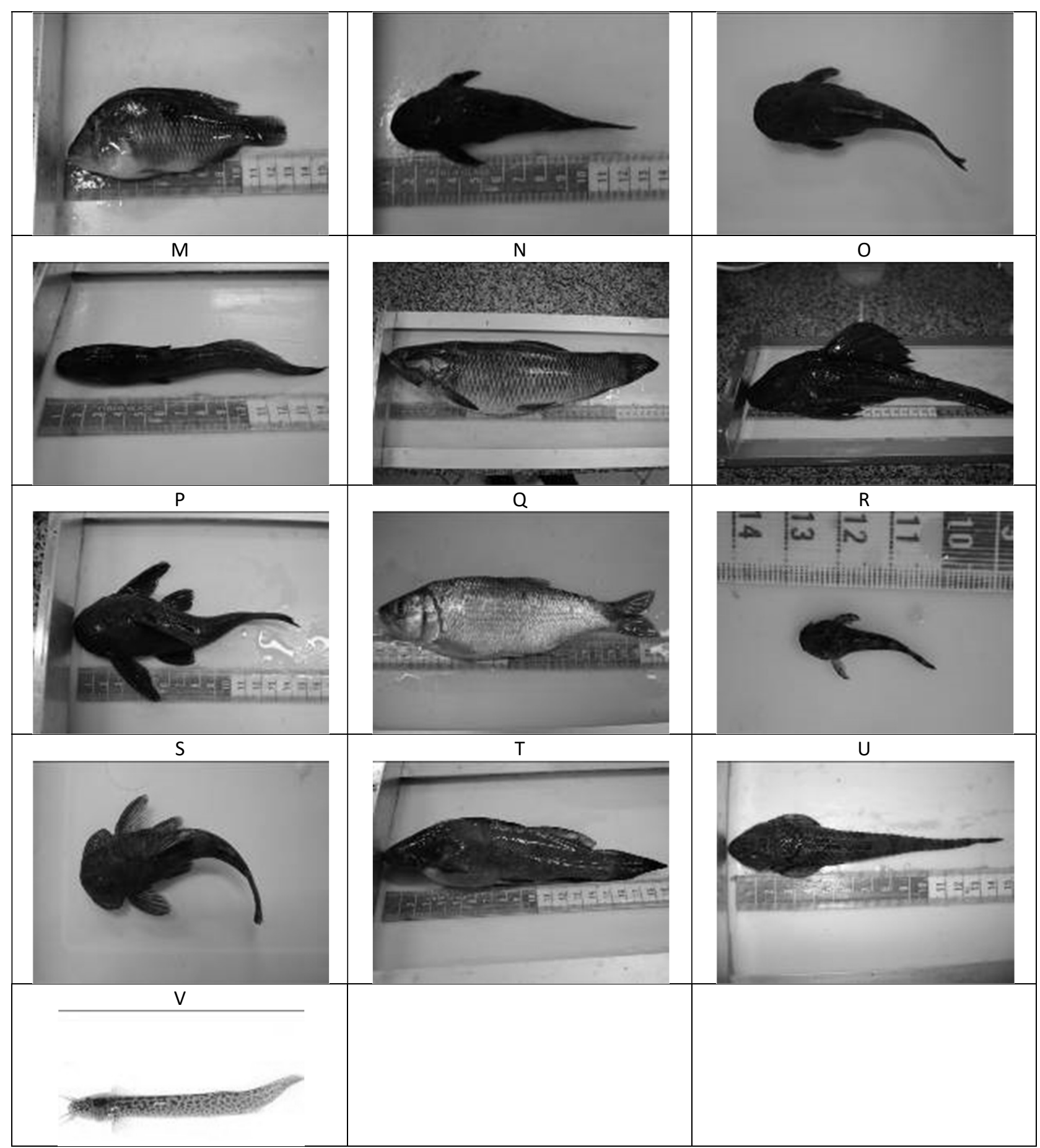

Figura 2: Espécies capturadas durante os monitoramentos ictiofaunisticos na área do Parque Nacional das Araucárias. Legenda: A (Astyanax bimaculatus), B (Astyanax scabripinnis), C (Ancistrus taunayi), D (Australoheros facetus), E (Bryconamericus stramineus), F (Crenicichla empheres). G (Crenicichla lepidota), H (Cyprinus carpio), I (Geophagus brasiliensis), J (Gymnogeophagus gymnogenys), K (Hemiancistrus meizospilos), L (Hemiancistrus sp.), M (Heptapterus mustelinus), N (Hoplias malabaricus), O (Hypostomus commersonii), P (Hypostomus aspilogaster), Q (Oligosarcus brevioris), R (Pareiorhamphis vestigipinnis), S (Pareiorhamphis steindachneri), T (Rhamdia quelen), U (Rineloricaria sp.) e V (Trichomycterus sp.).

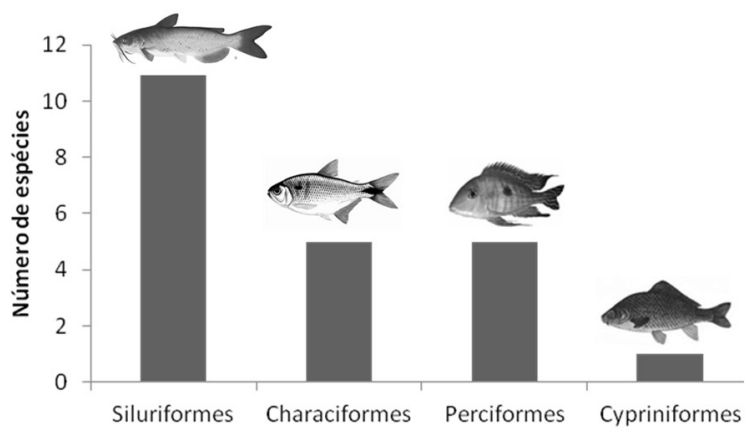

$$
\text { Ordens }
$$

Figura 3: Número de espécies para as ordens capturados durante as amostragens na área do PNA entre julho de 2011 e julho de 2012. 
As ordens Characiformes e Siluriformes, de forma geral, foram as mais amplamente distribuídas, enquanto que Perciformes e Cypriniformes foram registrados em poucos pontos amostrais. As espécies com maior distribuição na região do parque foram Astyanax scabripinnis, Oligossarcus brevioris, Hoplias malabaricus e Rhamdia quelen. O contrário percebeu-se para a maioria das espécies, com distribuição restrita a um ou dois cursos d'água (e. g. Ancistrus taunayi, Hypostomus aspilogaster, Heptapterus mustelinus, Crenicichla empheres, Australoeros facetum e Gymnogeophagus gymnogens) (Tabela 2).

Os pontos que apresentaram as maiores riquezas foram rio do Mato com 14 espécies, seguido do rio Chapecó e Chapecozinho com 12 e 13 espécies, respectivamente. Por outro lado, a menor riqueza foi registrada para Açude Adami e Riacho dos Morcegos com uma e três respectivamente. A curva de rarefação apresenta os valores referentes ao número de acumulação de espécies coletadas em cada uma das unidades amostrais ao longo das cinco amostragens. Percebeu-se que a curva de rarefação revelou tendência a assíntota, onde as espécies mais abundantes foram capturadas a partir das primeiras amostragens, enquanto que as tidas como raras no decorrer das demais campanhas (Figura 4).

Tabela 2. Espécies de peixes ocorrentes (assinalada por um X) nos pontos amostrados (P) sendo RCH - Rio Chapecó; RA - Riacho Ameixeira; RM - Riacho dos Morcegos; RCHZ - Rio Chapecozinho; CJ - Caratuva Jusante; BC - Barramento Caratuva; CM - Caratuva Montante RMT - Rio do Mato; AÇC - Açude Caratuva; AÇA - Açude Adami; no Parque Nacional das Araucárias, SC, entre julho/2011 e julho/2012.

\begin{tabular}{|c|c|c|c|c|c|c|c|c|c|c|c|}
\hline Táxon/Ponto & $\begin{array}{c}\text { P1 } \\
\text { RCH }\end{array}$ & $\begin{array}{l}\text { P2 } \\
\text { RA }\end{array}$ & $\begin{array}{l}\text { P3 } \\
\text { RM }\end{array}$ & $\begin{array}{c}\text { P4 } \\
\text { RCHZ }\end{array}$ & $\begin{array}{l}\text { P5 } \\
\text { CJ }\end{array}$ & $\begin{array}{l}\text { P6 } \\
\text { BC }\end{array}$ & $\begin{array}{l}\text { P7 } \\
\text { CM }\end{array}$ & $\begin{array}{c}\text { P8 } \\
\text { RMT }\end{array}$ & $\begin{array}{c}\text { P9 } \\
\text { AÇC }\end{array}$ & $\begin{array}{l}\text { P10 } \\
\text { AÇA }\end{array}$ & $\begin{array}{c}\text { Abundância } \\
\text { Total }\end{array}$ \\
\hline \multicolumn{12}{|l|}{ CHARACIFORMES } \\
\hline \multicolumn{12}{|l|}{ CHARACIDAE } \\
\hline Astyanax bimaculatus (Linnaeus, 1758) & $\mathrm{x}$ & & & $\mathrm{x}$ & & $\mathrm{x}$ & & $\mathrm{x}$ & $\mathrm{x}$ & & 31 \\
\hline Astyanax scabripinnis (Jenyns, 1842) & $\mathrm{x}$ & $\mathrm{x}$ & & $\mathrm{x}$ & $\mathrm{x}$ & $\mathrm{x}$ & $\mathrm{x}$ & $\mathrm{x}$ & $\mathrm{x}$ & & 305 \\
\hline Bryconamericus stramineus (Eigenmann, 1908) & $\mathrm{x}$ & & & & & & & $\mathrm{x}$ & & & 22 \\
\hline Oligosarcus brevioris (Menezes, 1987) & $\mathrm{x}$ & & & $\mathrm{X}$ & $\mathrm{x}$ & $\mathrm{x}$ & $\mathrm{x}$ & $\mathrm{X}$ & & & 135 \\
\hline \multicolumn{12}{|l|}{ ERYTHRINIDAE } \\
\hline Hoplias malabaricus (Bloch, 1794) & $\mathrm{x}$ & $\mathrm{X}$ & & $\mathrm{X}$ & & $\mathrm{x}$ & $\mathrm{x}$ & $\mathrm{X}$ & & $\mathrm{x}$ & 27 \\
\hline \multicolumn{12}{|l|}{ CYPRINIFORMES } \\
\hline \multicolumn{12}{|l|}{ CYPRINIDAE } \\
\hline Cyprinus carpio Linnaeus, 1758 & & & & & & & & & $\mathrm{X}$ & & 7 \\
\hline \multicolumn{12}{|l|}{ PERCIFORMES } \\
\hline \multicolumn{12}{|l|}{ CICHLIDAE } \\
\hline Crenicichla empheres (Lucena, 2007) & $\mathrm{x}$ & & & & & & & & & & 12 \\
\hline Crenicichla lepidota Heckel, 1840 & & & & & & $\mathrm{x}$ & & & & & 5 \\
\hline Gymnogeophagus gymnogenys (Hensel, 1870) & & & & $\mathrm{x}$ & & & & $\mathrm{x}$ & & & 9 \\
\hline Geophagus brasiliensis (Quoi e Gaimard, 1824). & & & & $\mathrm{x}$ & $\mathrm{x}$ & & & $\mathrm{x}$ & & & 146 \\
\hline Australoheros facetus (Jenyns, 1842) & & & & & & & & $\mathrm{X}$ & & & 3 \\
\hline \multicolumn{12}{|l|}{ SILURIFORMES } \\
\hline \multicolumn{12}{|l|}{ LORICARIIDAE } \\
\hline Ancistrus taunayi (Miranda Ribeiro, 1928) & $\mathrm{x}$ & & & $\mathrm{x}$ & & & & & & & 5 \\
\hline Hemiancistrus sp. & $\mathrm{x}$ & $\mathrm{x}$ & $\mathrm{x}$ & $\mathrm{x}$ & $\mathrm{x}$ & & & & & & 1 \\
\hline Hemiancistrus meizospilos (Cardoso \& da Silva, 2004) & & & & & & & & $\mathrm{x}$ & & & 25 \\
\hline Hypostomus commersonii (Valenciennes, 1836) & & & & $\mathrm{x}$ & & $\mathrm{x}$ & & $\mathrm{x}$ & & & 46 \\
\hline Hypostomus aspilogaster (Cope, 1894) & $\mathrm{x}$ & & & & & & & & & & 2 \\
\hline Pareiorhamphis vestigipinnis Perreira e Reis, 1992 & $\mathrm{x}$ & & & & & & & & & & 15 \\
\hline Pareiorhamphis steindachneri (Miranda \& Ribeiro, 1918) & $\mathrm{x}$ & $\mathrm{x}$ & $\mathrm{x}$ & $\mathrm{x}$ & $\mathrm{x}$ & & & & & & 116 \\
\hline Rineloricaria sp. & $\mathrm{x}$ & & & & & & & $\mathrm{x}$ & & & 17 \\
\hline \multicolumn{12}{|l|}{ HEPTAPTERDAE } \\
\hline Rhamdia quelen Quoi \& Gaimard, 1824 & $\mathrm{x}$ & & & $\mathrm{X}$ & $\mathrm{x}$ & $\mathrm{x}$ & $\mathrm{x}$ & $\mathrm{x}$ & $\mathrm{X}$ & & 65 \\
\hline Heptapterus mustelinus (Valenciennes, 1908) & & & & $\mathrm{x}$ & & & & $\mathrm{x}$ & & & 5 \\
\hline \multicolumn{12}{|l|}{ TRICHOMYCTERIDAE } \\
\hline Trichomycterus sp. & & $\mathrm{X}$ & $\mathrm{x}$ & & & & & $\mathrm{x}$ & & & 23 \\
\hline Riqueza & 13 & 5 & 3 & 12 & 6 & 7 & 4 & 14 & 4 & 1 & \\
\hline
\end{tabular}




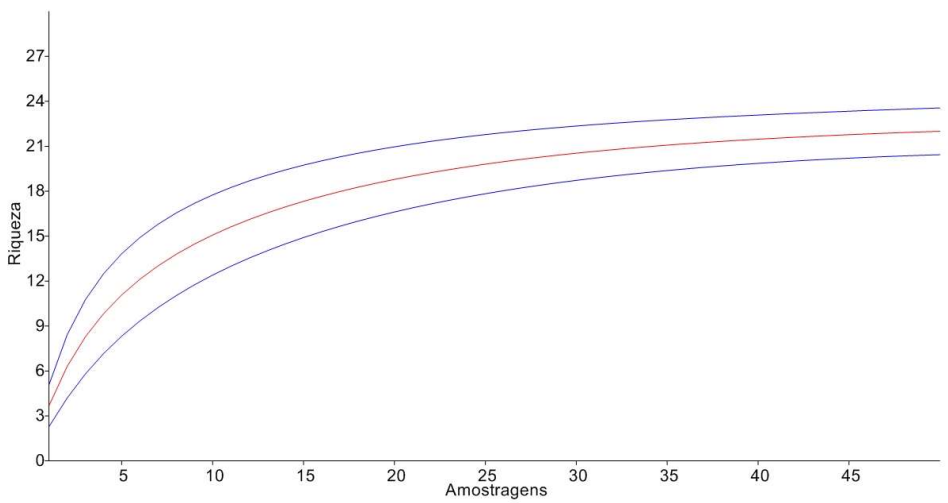

Figura 4: Curva de Rarefação (Média em vermelho e Desvio Padrão em azul) referente às unidades amostrais para coleta de peixes na área do PNA.

\section{DISCUSSÃO}

Estudos recentes revelam que o número de espécies de peixes encontradas para a Mata Atlântica varia entre 270 a 350, distribuída ao longo de sua área que originalmente estendia-se do Rio Grande do Norte até o Rio Grande do Sul (VARI et al., 1998; MIRANDA, 2012). Atualmente, menos de 7\% dessa área pode ser encontrada, onde em algumas extensões foram criadas Unidades de Conservação a fim de manter o pouco que restou desse bioma, preservando sua fauna e flora (MYERS et al., 2000). Uma destas áreas no estado de Santa Catarina é o Parque Nacional das Araucárias, a qual nenhum estudo havia sido realizado acerca da sua fauna íctica.

A composição da assembleia de peixes encontrada para a região do PNA variou de $6 \%$ a $8 \%$ do total registrado para a Floresta Atlântica. Resultado semelhante foi encontrado por Copatti et al. (2011) para o rio Cambará com 26 espécies e por Casatti et al. (2001) na área do Parque Estadual Morro dos Diabos, com o registro de 22 espécies. De acordo Azevedo et al. (2003), Pessano et al. (2005), Kaufmann et al. (2009) e Amaral et al. (2010), pequenos rios e riachos da bacia do rio Uruguai apresentam riqueza entre 20 a 50 espécies, com alguns endemismos e presença de exóticas, o que corrobora quanto ao registro de espécies neste estudo.

Estudos relacionados a assembleias de peixes envolvem uma variedade de parâmetros mensuráveis, em que o número de ordens, famílias e espécies são medidas interessantes a serem observadas (MATTHEWS, 1998). Grande parte dos trabalhos realizados para a região Neotropical apresenta a ordem Characiformes com o maior número de espécies, seguido de Siluriformes (APONE et al., 2008; CASTRO et al., 2003; AZEVEDO et al., 2003). No entanto, para a área estudada foi registrado aproximadamente $55 \%$ a mais de Siluriformes em relação aos Characiformes, corroborando com os resultado de Fogaça et al. (2003) em estudos realizados para o rio do Quebra em Antonina/Paraná e Bifi et al. (2006) no rio dos Padres na bacia do rio Iguaçu. De acordo com Wolff (2012), a maior presença de Siluriformes pode estar relacionada à presença de cascudos de pequeno porte, que apresentam pré-adaptações para ambientes de cabeceira, com fluxo maior de corredeiras. No entanto, para a região Neotropical, Castro (1999) e Lowe-McConnell (1999) revelam que o predomínio de Characiformes e Siluriformes em riachos não é característico para todos os ambientes, podendo haver variações de riqueza entre eles conforme o estado de preservação dos ambientes e de suas 
características ambientais e estruturais.

O registro de uma espécie reconhecida como exótica e/ou invasora (Cyprinus carpio), demonstra a condição do ambiente, haja vista que a área da UC tinha até recentemente diferentes usos no seu interior. A espécie é frequentemente encontrada em rios e reservatórios sendo oriundas de introduções acidentais (SEVERI et al., 1994) e intencionais a partir de atividades de piscicultura e 'peixamento' de lagos. A carpa Cyprinus carpio tem uma antiga história de introduções no Brasil, ocorrendo no país desde o final do século XIX (AGOSTINHO et al., 1996) e conforme a IUNC (2010) é considerada uma das 100 piores espécies invasoras do mundo, e seus impactos ultrapassam os limites dos ambientes, pois afetam diretamente aspectos econômicos e sociais. Quando introduzida na comunidade de peixes, esta espécie pode causar forte alteração junto à assembleia, devido à predação de ovos e juvenis e a competição, diminuindo a população de espécies nativas e com valor econômico ou em casos extremos podendo levar ao extermínio de espécies (FULLER et al., 1999; ELVIRA, 2001; ESPÍNOLA et al., 2007). Isso demonstra que a presença de Cyprinus carpio dentro da área do PNA pode ser considerada uma ameaça, entretanto, foi restrito ao açude Adami, não sendo registrado nos ambientes lóticos. Mas, devido sua característica invasora, há a necessidade de implantação de um plano de manejo com intuito de diminuir o risco de sua introdução nos demais ambientes ou mesmo erradicá-la.

A interferência nos ambientes aquáticos seja ela causada pelo uso do seu entorno, introdução de espécies exóticas, poluentes ou alteração do seu curso natural, principalmente com a finalidade de irrigação ou construção de barragens vem alterando as comunidades ícticas, afetando com maior rapidez espécies endêmicas ou com população menores. No ano de 2007, duas novas espécies de Crenicichla foram descrita para o rio Chapecó, sendo as mesmas consideradas endêmicas da bacia e com registros apenas para a região do município de Abelardo Luz, Santa Catarina (LUCENA, 2007). Dentre estas, Crenicichla empheres foi registrada neste estudo para o mesmo rio Chapecó, contudo, entre as cinco incursões realizadas, apenas 12 indivíduos foram capturados, corroborando com os resultados registrados pelo autor acima citado, que em sua pesquisa registrou apenas 27 espécimes, que evidenciou sua pequena população para este curso hídrico. A espécie consta atualmente na lista de ameaças de extinção para o estado de Santa Catarina (IUCN, 2011), contudo sua população apresenta-se dividida, pois recentemente foi construída uma Pequena Central Hidrelétrica $(\mathrm{PCH})$ a montante da área do PNA impossibilitando que a população de jusante tenha contato com a localizada acima do reservatório, sendo importante a preservação destes hábitats para que a espécie possa manter e aumentar sua população e dispersão dentro da bacia.

A presença de espécies ainda não registradas ou descritas para a bacia do alto rio Uruguai, vem aumentando de acordo com número crescente de estudos. Fato este foi evidenciado com a captura de três espécies, Crenicichla lepidota com ocorrência anterior para a parte baixa e média desta mesma bacia, Pareiorhamphis vestigipinnis para o rio Canoas e seus afluentes e Pareiorhamphis steindachneri para a região leste do estado nos rios Cubatão e Itajaí-Açu (REIS, 2003). Acredita-se que o registro tardio destas espécies para a bacia do alto rio Uruguai seja decorrente dos poucos estudos realizados para cursos hídricos menores, tendo em vista maiores interesses para o canal principal do rio Uruguai. 
Ao tratar da distribuição de espécies dentro da área do PNA, nota-se que o predomínio de Characiformes e Siluriformes está relacionado a heterogeneidade ambiental. Segundo Lowe-McConnel (1999) e Castro et al. (1999) Characiformes e Siluriformes são facilmente encontrados nos mais diversos ambientes (e. g. lóticos e lênticos) principalmente os que apresentam tamanhos reduzidos tendo assim maior facilidade de ajustar-se em distintos habitats. Ademais, os Perciformes, têm por preferência ocupar ambientes de águas calmas, característica contrária a encontrada na maior parte dos riachos e rios que fazem parte da rede hídrica da Floresta Atlântica (LANGEANI et al., 2007; WOLFF, 2012).

A maior distribuição de Astyanax scabripinnis na área de abrangência do PNA (e. g. riachos, pequenos rios, reservatório e lagos artificiais) demonstra sua plasticidade trófica, sendo esta considerada uma espécie generalista e oportunista, ou seja, fazem uso dos mais variados recursos alimentares que os diferentes ambientes proporcionam (HAHN et al., 2007). A presença desta espécie, pode auxiliar para explicar o fato da maior distribuição de Hoplias malabaricus, Rhamdia quelen e Oligosarcus brevioris, as quais são consideradas predadoras, não essencialmente carnívoras, que além de outros recursos como insetos e vegetais, alimentam-se de peixes de pequeno porte, tais como Astyanax scabripinnis, Astyanax bimaculatus e Bryconamericus stramineus (HERMES-SILVA et al., 2004; GAELH et al., 1998; GOMIEIRO et al., 2007).

A maioria das espécies apresentou baixa frequência de ocorrência, sendo registradas em poucos pontos amostrais e com algumas exclusividades. Conforme Castro (1999) essas exclusividades (e. g. Pareiorhamphis vestigipinnis, Crenicichla empheres e Australoheros facetus) podem estar relacionadas a características como baixa abundância e exigências específicas a determinados ambientes, preferencialmente sob baixa pressão externa. Além disso, segundo o autor, estas espécies geralmente apresentam tamanho reduzido, estratégias de vida como baixa fecundidade ou com desova parcelada durante todo o ano. Está baixa fecundidade para algumas espécies é recompensada por outras características, como o maior tamanho dos ovos que ao eclodir possibilitarão as larvas, também maiores, a explorar mais facilmente o ambiente (Braga et al., 2007), como evidenciado em um estudo realizado por Mazzoni et al. (2002) com G. brasiliensis, onde para cada habitat diferente, havia variação na fecundidade e tamanho dos ovos. Isso tudo ressalta a importância de preservar tais ambientes (cabeceiras) com intuito de manter a comunidade íctica na área do PNA, bem como de toda bacia do rio Chapecó.

De acordo com a teoria do contínuo fluvial, trechos intermediários de cursos hídricos tendem a registrar maior riqueza (VANNOTE et al., 1980) podendo isso estar relacionada a algumas características físicas dos ambientes, provido de corredeiras e remansos, fundo repleto de pedras e com margens protegidas pela vegetação, propiciando um ambiente com potencial alimentar, reprodutivo e protetor, corroborando com o registrado para esta pesquisa, onde para o rio do Mato encontrou-se o maior número de espécies. Segundo Martin-Smith (1998) a diversidade de hábitats influencia na diversidade de recursos, ocupação e permanência das espécies, onde locais com tais características tendem a apresentar uma assembleia íctica mais heterogênea.

A tendência à assíntota registrada pela rarefação do número de espécies e suas respectivas abundâncias revelou que a assembleia íctica na área do PNA aproximou-se do número total esperado. Santos 
(2003) relata que uma das prováveis causas da não estabilização da curva pode estar relacionada à frequência de captura de espécies raras ou não frequentes. No entanto, segundo Uieda et al. (1999) a dificuldade em capturar todas as espécies de peixes de um ambiente pelos métodos de captura traz a incerteza de uma amostragem satisfatória, onde o ideal seria um período maior de estudo. Além disso, as espécies dominantes foram capturadas a partir da primeira coleta e as espécies reconhecidas como raras, foram coletadas entre as demais. Isso pode estar relacionado à variação das condições ambientais entre as campanhas, haja vista que as espécies possuem características distintas, com maior atividade em algumas épocas do ano (BIFI et al., 2006). No entanto, seria necessário um estudo em longo prazo a fim de capturar todas, ou a maior parte das espécies de peixes do PNA e assim, estabilizar por completo a curva de rarefação.

\section{CONCLUSÕES}

Os resultados encontrados neste estudo, revelam basicamente que a ictiofauna presente no interior do Parque Nacional das Araucárias segue um mesmo padrão de composição quando comparados a outras regiões de abrangência da Mata Atlântica, onde em sua maioria as espécies possuem tamanhos diminutos e possíveis dependências ambientais para sua existência. Tal indicação reforça a necessidade de preservação de remanescentes deste bioma, a fim de proteger e manter a rica diversidade de peixes encontrados especialmente em pequenos cursos d'água. Ademais, espera-se que este trabalho possa auxiliar futuras pesquisas na área do Parque Nacional das Araucárias e na região da bacia do alto rio Uruguai.

\section{REFERÊNCIAS}

AGOSTINHO, A. A.; JÚLIO JÚNIOR, H. F.. Ameaça ecológica: peixes de outras águas. Ciência Hoje, v.21, p.36-44, 1996.

AMARAL, C.; BARP, E. A.. Ictiofauna do rio Engano nos municípios de Irani, Ipumirim e Itá em Santa Catarina, sul do Brasil. Biotemas, Florianópolis, v.23, p.147-152, 2010.

APONE, F.; OLIVEIRA, A. K.; GARAVELLO, J. C.. Composição da ictiofauna do rio Quilombo, tributário do rio Mogi-Guaçu, bacia do alto rio Paraná, sudeste do Brasil. Biota Neotrópica, v.8, p.93-107, 2008.

AZEVEDO, C.; PESSANO, E.; TOMASSONI, D.; QUEROL, M. V.; QUEROL, E.. Diversidade específica, densidade e biomassa da ictiofauna da nascente do arroio Felizardo, bacia do rio Uruguai Médio, Uruguaiana/RS, Brasil. Biodiversidade Pampeana, v.1, n.1, p.35-45, 2003.

BARRETO, A. P.; ARANHA, J. M. R.. Assembleia de peixes de um riacho da Floresta Atlântica: composição e distribuição espacial (Guaraqueçaba, Paraná, Brasil). Acta Sci. Biol. Sci. v.27, n.2, p.153-160, 2005.

BIFI, A. G.; BAUMGARTNER, D.; BAUMGARTNER, G.; FRANA, V. A.; DEBONA, T.. Composição especifica e abundância da ictiofauna do rio dos Padres, bacia do rio Iguaçu, Brasil. Acta Scientiarum Biological Sciences, Maringá, v.28, n.3, p.203211, 2006.

BUCKUP, P. A.; MENEZES, N. A.; GHAZZI, M. S.. Catálogo das espécies de peixes de água doce do Brasil. Rio de Janeiro,
Museu Nacional, 2007.

CASATTI, L.; CASTRO, R. M. C.; LANGEANI, F.. Peixes de riacho do Parque Estadual Morro do Diabo, bacia do alto rio Paraná, SP. Biota Neotrópica, São Paulo, v.1, n.1, p.1-15, 2001.

CASTRO, R. M. C.. Evolução da ictiofauna de riachos sulamericanos: padrões gerais e possíveis processos causais. In: CASTRO, R. M. C.. Ecologia de Peixes de Riachos: Estado Atual e Perspectivas. Ecologia Brasiliensis. 1999, p.139-155.

CASTRO, R. M. C.; CASATTI, L.; SANTOS, H. F.; FERREIRA, K. M.; RIBEIRO, A. C.; BENINE, R. C.; DARDIS, G. Z. P.; MELO, A. L. A.; STOPIGLIA, R.; ABREU, T. X.; BOCKMANN, F. A.; CARVALHO, M.; GIBRAN, F. Z.; LIMA, F. C. T.. Estrutura e composição da ictiofauna de riachos do rio Paranapanema, sudeste e sul do Brasil. Biota Neotrópica, São Paulo, v.3, n.1, p.1-31, 2003.

CETRA, M.; SARMENTO-SOARES, L. M.; MARTINS-PINHEIRO, R. F.. Peixes de riachos e novas Unidades de Conservação no Sul da Bahia. Pan-American Journal of Aquatic Sciences, v.5, p.11-21, 2010.

COPATTI, C. E.; COPATTI, B. R.. Variação sazonal e diversidade de peixes do rio Cambará, Bacia do rio Uruguai. Biota Neotrópica, v.11, p.265-271, 2011.

ELVIRA, B.. Identification of non-native freshwater fishes established in Europe and assessment of their potential 
threats to the biological diversity. In: CONVENTION ON THE 57 CONSERVATION OF EUROPEAN WILDLIFE AND NATURAL HABITATS, COUNCIL OF EUROPE, WORKING DOCUMENT TPVS, 6. Anais. 2001. p.35.

ESPÍNOLA, L. A.; JULIO, H. F.. Espécies invasoras: conceitos, modelos e atributos. Interciência, v.32, n.3, p.580-585, 2007.

FOGAÇA, F. N. O.; ARANHA, J. M. R.; ESPER, M. L. P.. Ictiofauna do rio do Quebra (Antonina, PR, Brasil): ocupação espacial e hábito alimentar. Interciência, v.28, n.3, p.168173, 2003.

FULLER, P. L.; NICO, L.G.; WILLIAMS, J.D. Nonindigenous Fishes Introduced into Inland Water of the United States. Bethesda: American Fisheries Society Special Publication, 1999.

GEALH, A. M.; HAHN, N. S.. Alimentação de Oligosarcus longirostris Menezes \& Gèry (Osteichthyes, Acestrorhynchinae) do reservatório de Salto Segredo, Paraná, Brasil. Revista Brasileira de Zoologia, v.15, n.4, p.985-993, 1998.

GOMIEIRO, M.; SOUZA, U. P.; BRAGA, F. M. S.. Reprodução e alimentação de Rhamdia quelen (Quoy e Gaimard, 1824) em rios do núcleo de Santa Virgínia Parque Estadual Serra do Mar, São Paulo, SP. Biota Neotrópica, v.7, n.3, p.127-133, 2007.

GOTELLI, N. J.; COLWELL, R.. Quantifying biodiversity: procedures and pitfalls in the measurement and comparison of species richness. Ecol. Lett., v.4, p.379-391, 2001.

GRAÇA, W. J.; PAVANELLI, C. S.. Peixes da planície de inundação do alto rio Paraná e nas áreas adjacentes. Maringá: EDUEM, 2007.

HAHN, N. S.; FUGI, R.. Alimentação de peixes em reservatórios brasileiros: alterações e consequências nos estágios iniciais do represamento. Oecologia brasiliensis, v.11, n.4, p.469-480, 2007.

HERMES-SILVA, M. S.; ZANIBONI FILHO, E.. Biologia alimentar e reprodutiva do peixe cachorro (Oligosarcus jenynsii Gunther, 1864) na região do alto rio Uruguai. Acta Sci. Biol. Sci., v.26, n.2, p.175-179, 2004.

HICKSON, R. G. et al. Métodos para a caracterização da ictiofauna em estudos ambientais: Ictiofauna de microbacia do rio Jaguari/RS, Brasil. Biota Neotrópica, v.9, n.2, p.179186, 2009. DOI: http://dx.doi.org/10.1590/S1676$\underline{06032009000200017}$

\section{IUCN. IUCN Red List of Threatened Species. 2011.}

\section{KLEIN, R. M.. Mapa Fitogeográfico do Estado de Santa} Catarina. Flora llustrada Catarinense, 1978.

KAUFMANN, V.; PINHEIRO, A.. Relações entre diversidade íctica e fatores hidrodinâmicos de um riacho na Bacia do Rio Uruguai. Biota Neotrópica, v.9, n.1, p.47-53, 2009.

LANGEANI, F.; CASTRO, R. M. C.; OYAKAWAI, O. T.; SHIBATTA, O. A.; PAVANELLI, C. S.; CASATTI, L.. Diversidade da ictiofauna do Alt Rio Paraná: Composição atual e perspectivas futuras. Biota Neotrópica, v.7, n.3, p.181-197, 2007.

LOWE-MCCONNELL, R. H.. Estudos ecológicos em comunidades de peixes tropicais. São Paulo: EDUSP, 1999.

LUCENA, C. A. S.. Two new species of the genus Crenicichla Henckel, 1940 from the upper rio Uruguay drainage (Perciformes: Ciclidae). Neotropical Ichthyology, v.5, p.449456, 2007.

MARTIN-SMITH, K. M.. Relationships between fishes and habitat in rainforest streams in Sabah, Malaysia. Journal of Fish Biology, v.52, n.3, p.458-482, 1998. Dol: https://doi.org/10.1111/j.1095-8649.1998.tb02010.x

MATTHEWS, W. J. Patterns in Freshwater Fish Ecology. New York: Chapman and Hall, 1998.

MENEZES, N. A.; WEITZMAN, S. H.; OYAKAWA, O. T.; LIMA, F. C. T.; CASTRO, R. M. C.; WEITZMAN, M. J.. Peixes de água doce da Mata Atlântica: lista preliminar das espécies e comentários sobre conservação de peixes de água doce neotropicais. São Paulo: Museu de Zoologia - Universidade de São Paulo, 2007.

MYERS, N.; MITTERMEIER, R. A.; MITTERMEIER, C. G.; FONSECA, G. A. B.; KENT, J.. Biodiversity hotspots for conservationpriorities. Nature, v.403, p.853-845, 2000.

MIRANDA, J. C.. Ameaças aos peixes de riachos da Mata Atlântica. Natureza online, v.10, n.3, p.136-139, 2012.

MMA. Ministério do Meio Ambiente. Plano de manejo: Parque Nacional das Araucárias. Brasília: MMA/SBF, 2010

OYAKAMA, O. T.; AKAMA, A.; MAUTARI, K. C.; NOLASCO, J. C.. Peixes de riacho da Mata Atlântica. São Paulo: Neotropical, 2006.

PESSANO, E. F. C.; AZEVEDO, C. L. O.; QUEROL, M. V. M.; QUEROL, E.; BRASIL, L. G.; CASTRO, L. R. B.; PINTO, T. B.; CORRÊA, F. V.. Ictiofauna do arroio Quarai-Chico, Bacia do médio rio Uruguai, no interior do Parque Estadual do Espinilho, Rio Grande do Sul, Brasil. Biotemas, v.18, n.2, p.143-153, 2005.

REIS, R. E.; KULLANDER, S. O.; FERRARIS Jr, C. J.. Check list of the freshwater fishes of South and Central America. Porto Alegre: Edipucrs, 2003.

SANTA CATARINA. Secretaria de Estado do Desenvolvimento Sustentável. Panorama dos Recursos Hídricos de Santa Catarina. Florianópolis, 2007.

SANTOS, A. J.. Estimativas de riqueza em espécies. In: CULLEN JR, L.; VALLADARES-PADUA, C.; RUDRAN, R.. Métodos de estudos em biologia da conservação \& manejo da vida silvestre: 19-41. Curitiba: UFPR, 2003. p.19-41.

SCHAEFER, S. A.. Conflict and resolution: Impact of new taxa on phylogenetic studies. In: SCHAEFER, S. A.. Ciência Tecnológica, Porto Alegre: PUCRGS, 1998.

SEVERI, W.; CORDEIRO, A. A. M.. Catálogo de peixes da bacia do rio Iguaçu. Curitiba: IAP/GTZ, 1994. 
UIEDA, V. S.; CASTRO, R. M. C.. Coleta e fixação de peixes de riachos. In: CARAMASCHI, E. P.; MAZZONI, R.; PERES-NETO, P. R.. Ecologia de Peixes de Riachos, Série Oecologia Brasiliensis. 6 ed. Rio de Janeiro: PPGE-UFRJ, 1999. p.1-22.

VANNOTE, R. L; MINSHALL, G. W.; CUMMINS, K. W.; SEDELL, J. R.; CUSHING, C. E.. The river continuum concept. Canadian Journal of Fishery and Aquatic Science, v.37, n.1, p.130-137, 1980.

VARI, R. P.; MALABARBA, L. R.. Neotropical ichthyology: an overview. In: VARI, R. P.; MALABARBA, L. R.. Phylogeny and classification of Neotropical fishes. Porto Alegre: Edipucrs, 1998.

WOLF, L. L.. Assembleia de peixes de um riacho da encosta Atlântica, Brasil: estrutura espacial, uso dos recursos alimentares e relação ecomormológica. Tese (Doutorado em Ecologia de Ambientes Aquáticos Continentais) Universidade Estadual de Maringá, 2012.

ZANIBONI FILHO, E.; MEURER, S.; SHIBATTA, O. A.; NUÑER, A. P. O.. Catálogo ilustrado de peixes do Alto rio Uruguai. Florianópolis: UFSC, 2004.

A CBPC - Companhia Brasileira de Produção Científica (CNPJ: 11.221.422/0001-03) detém os direitos materiais desta publicação. Os direitos referem-se à publicação do trabalho em qualquer parte do mundo, incluindo os direitos às renovações, expansões e disseminações da contribuição, bem como outros direitos subsidiários. Todos os trabalhos publicados eletronicamente poderão posteriormente ser publicados em coletâneas impressas sob coordenação da Sustenere Publishing, da Companhia Brasileira de Produção Científica e seus parceiros autorizados. Os (as) autores (as) preservam os direitos autorais, mas não têm permissão para a publicação da contribuição em outro meio, impresso ou digital, em português ou em tradução. 